\title{
Deciphering the Role of Insulin-Like Growth Factor-I Receptor in Trastuzumab Resistance
}

\author{
Rita Nahta \\ Departments of Pharmacology, Hematology and Medical Oncology, School of Medicine; Winship Cancer Institute; \\ Molecular and Systems Pharmacology Program, Graduate Division of Biological and Biomedical Sciences, Emory University, \\ Suite 5001, 1510 Clifton Road, Atlanta, GA 30322, USA
}

Correspondence should be addressed to Rita Nahta, rnahta@emory.edu

Received 25 April 2012; Accepted 19 June 2012

Academic Editor: Sue S. Yom

Copyright ( 2012 Rita Nahta. This is an open access article distributed under the Creative Commons Attribution License, which permits unrestricted use, distribution, and reproduction in any medium, provided the original work is properly cited.

\begin{abstract}
Resistance to the HER2-targeted antibody trastuzumab is a major clinical concern in the treatment of HER2-overexpressing metastatic breast cancer. Increased expression or signaling of the insulin-like growth factor-I receptor (IGF-IR) has been reported in a subset of cell lines and clinical samples derived from trastuzumab-resistant breast cancers. Genetic and pharmacologic inhibition of IGF-IR signaling has been shown to improve response to trastuzumab in trastuzumab-naïve and trastuzumabresistant models. In this paper, we will discuss the role of IGF-IR signaling in trastuzumab resistance. Further, we will discuss cotargeting IGF-IR and HER2 as a potential therapeutic strategy for HER2-over-expressing breast cancers that have progressed on trastuzumab treatment.
\end{abstract}

\section{Introduction}

Trastuzumab (Herceptin; Genentech, San Francisco, CA) is a humanized monoclonal antibody against an epitope in the extracellular domain of the HER2 receptor tyrosine kinase protein [1]. HER2 is overexpressed, generally due to amplification of the her 2 gene, in approximately $20-30 \%$ of human metastatic breast cancers (MBC), and is associated with reduced disease-free survival [2]. Trastuzumab effectively elicits pathologic complete responses in a large percentage of patients with HER2-positive MBC [3,4], particularly when combined with chemotherapy [5-7]. However, some patients do not respond to trastuzumab [3-7], displaying socalled primary, de novo, or intrinsic resistance. In addition, median duration of response to trastuzumab-based therapy was reported to be less than one year in initial trials [37], indicating that acquired resistance is a common clinical concern. A clearer understanding of the mechanisms that contribute to trastuzumab resistance is needed in order to develop new therapeutic strategies, and, ultimately, to improve survival outcomes for patients with HER2-overexpressing breast cancer.

\section{Preclinical Studies Examining IGF-IR and Trastuzumab Resistance}

Multiple molecular mechanisms driving trastuzumab resistance have been proposed. These mechanisms and potential treatment strategies to overcome resistance are discussed in detail in several recent and outstanding reviews [812]. In this paper, we will focus on one of the proposed mechanisms of trastuzumab resistance: increased signaling from the insulin-like growth factor-I receptor (IGF-IR).

$\mathrm{Lu}$ et al. [13] first provided data to support a possible role for IGF-IR in trastuzumab resistance. The authors [13] showed that trastuzumab effectively activated a G1 arrest response in the HER2-over-expressing breast cancer cell line SKBR3, which expresses a relatively low level of IGFIR. However, stable over-expression of IGF-IR prevented the trastuzumab-mediated growth arrest response in SKBR3 cells. A separate report [14] confirmed these findings by showing that $10 \mu \mathrm{g} / \mathrm{mL}$ trastuzumab reduced proliferation of SKBR3 cells by $25 \%$, while proliferation of SKBR3/IGF-IR stable transfectants was unaffected by as much as $60 \mu \mathrm{g} / \mathrm{mL}$ of trastuzumab. Under anchorage-independent conditions, 
trastuzumab inhibited colony growth of SKBR3 empty vector control cells by $62 \%$ versus only $12 \%$ inhibition in SKBR3 IGF-IR stable transfectants [13]. In addition, MDA231 breast cancer cells transfected with HER2 showed downregulation of IGF-IR and increased sensitivity to trastuzumab [15]. When these MDA231/HER2 cells were chronically exposed to trastuzumab, resistant clones developed. These MDA231/HER2 trastuzumab-resistant cells showed 3-fold higher expression of IGF-IR versus the MDA231/HER2 cells [15]. In addition, imaging studies showed that radiolabeled IGF-I could identify HER2-positive breast cancer cells that were resistant to trastuzumab and overexpressed IGF-IR [15], suggesting that IGF-IR expression may be used as a proxy to identify resistant cancers.

These preclinical studies led to the concept that IGF-IR over-expression may be associated with reduced response to trastuzumab. In support of this concept, Jerome et al. [16] examined IGF-IR levels in a cell line that had acquired resistance to trastuzumab. IGF-IR expression was up-regulated by approximately 3-fold in BT474 trastuzumab-resistant cells relative to the trastuzumab-sensitive parental BT474 line. Thus, chronic exposure to trastuzumab may result in upregulation of IGF-IR in association with resistance. In addition to acquired resistance, IGF-IR expression may be linked to intrinsic resistance to trastuzumab. Phospho-proteomic analysis was performed on the JIMT-1 HER2-positive cell line, which shows primary resistance to trastuzumab [17]. This analysis established that IGF-IR was constitutively activated in this cell line. Activated IGF-IR was localized primarily at focal adhesion structures within the cells.

We have also analyzed our models of acquired trastuzumab resistance to determine if IGF-IR was overexpressed. Resistant cells were developed by chronically culturing SKBR3 cells in trastuzumab and isolating resistant cells [18, 19]. In comparison to the parental SKBR3 cells, acquired trastuzumab-resistant SKBR3 cells did not show increased IGF-IR expression [20]. However, we identified a novel complex formation between IGF-IR and HER2 in resistant cells, which was not detected in trastuzumab-sensitive parental cells. Huang et al. [21] confirmed this IGF-IR/HER2 interaction and showed that HER3 is also found in this receptor complex. Thus, unique protein-protein interactions occur between HER2 and other receptors, including IGFIR and HER3, in cells with trastuzumab resistance. Our work showed that this unique interaction facilitates crosstalk from IGF-IR to HER2, such that IGF-I stimulation not only induces phosphorylation of IGF-IR, but also activates phosphorylation of HER2 [20]. IGF-I stimulation in the presence of an IGF-IR kinase inhibitor blocked this crosstalk [20], confirming that the crosstalk occurs from IGF-IR to HER2 and not through another mechanism. Further support for the concept of IGF-IR/HER2 crosstalk in trastuzumab-resistant cells was obtained by blocking IGFIR with monoclonal antibody alpha IR3 (Figure 1). When resistant cells were treated with alpha IR3 for 1 hour, total IGF-IR levels were unaffected [20]. However, IGF-IR expression was down-regulated when resistant cells were cultured with alpha IR3 overnight (Figure 1). In association with reduced IGF-IR, phosphorylation of HER2 was also

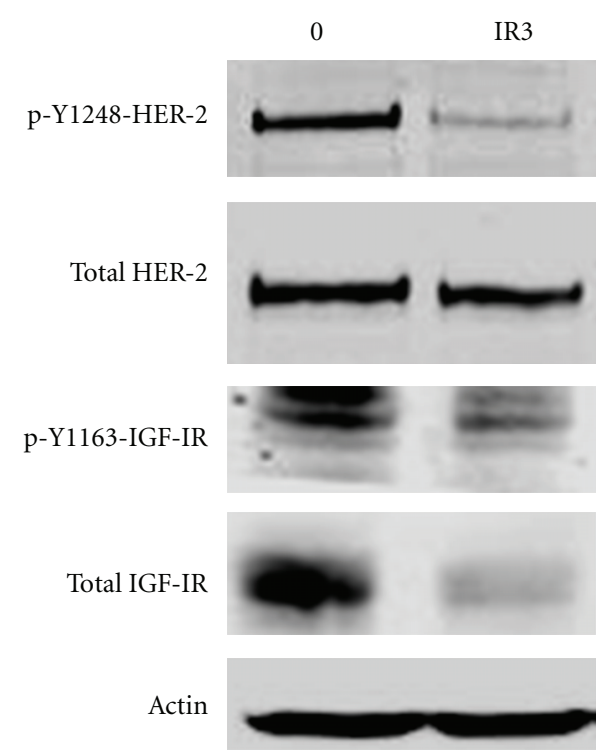

FIGURE 1: IGF-IR blockade reduces phosphorylation of HER2 in trastuzumab-resistant cells. SKBR3 cells with acquired resistance to trastuzumab were untreated or treated with $0.25 \mu \mathrm{g} / \mathrm{mL}$ alpha IR3 IGF-IR monoclonal antibody overnight. Total protein lysates were western blotted for phosphorylated and total HER2 and IGF-IR. Actin served as a loading control.

suppressed, although total HER2 levels were unaltered. Thus, stimulation or inhibition of IGF-IR resulted in an induction or suppression of HER2 phosphorylation, respectively, in cells with acquired trastuzumab resistance. These data are in direct support of IGF-IR crosstalk to HER2 in the context of trastuzumab resistance.

\section{Clinical Studies Examining IGF-IR and Trastuzumab Resistance}

Despite intriguing preclinical results suggesting a role for IGF-IR in trastuzumab resistance, the translational significance of IGF-IR over-expression or crosstalk to HER2 remains unclear. In two clinical correlative studies, no association was found between IGF-IR expression alone and trastuzumab response [22, 23]. However, increased IGF-IR expression plus increased downstream mTOR signaling correlated significantly with reduced response to trastuzumab [23]. Further, in a recent multivariate analysis [24], high IGFIR expression was associated with poor prognosis specifically in the HER2-positive subtype of breast cancers. Harris et al. [25] found a correlation between high IGF-IR expression and poor response to preoperative trastuzumab plus chemotherapy. Increased IGF-IR membrane staining, measured by IHC, was associated with lower response to preoperative trastuzumab plus vinorelbine, with a $50 \%$ median response rate in the high IGF-IR group versus $97 \%$ in the low IGFIR group [25]. Gallardo et al. [26] performed IHC for several proteins on tumor tissues obtained from patients with early stage or metastatic HER2-positive breast cancer treated with trastuzumab. Amongst 67 patients with early 


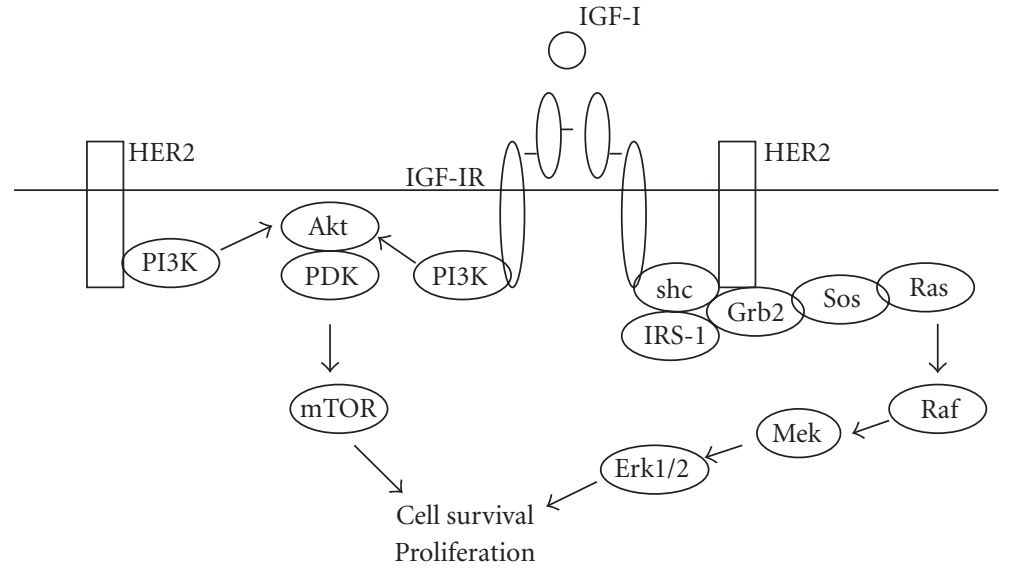

FIGURE 2: IGF-IR signaling pathway. Increased expression of IGF-IR, or IGF-IR interaction and crosstalk with HER2 have been reported in models of Herceptin resistance. PI3K signaling has been implicated as a potential mechanism of IGF-IR-mediated Herceptin resistance. Inhibition of IGF-IR has been shown to increase sensitivity to Herceptin in preclinical studies.

stage disease, IGF-IR over-expression or phosphorylation (inactivation) of the pro-apoptotic protein Bad correlated significantly with shorter progression-free survival (PFS). Amongst 75 patients with metastatic disease, decreased PFS correlated with increased PI3K signaling, and overall survival correlated with vascular invasion and EGFR over-expression. For all samples, staining of IGF-IR was high in $25 \%$ of tumors $(34 / 138)$, which was significantly associated $(P$-value less than or equal to 0.005$)$ with high grade, high mitotic index, and vascular invasion [26]. Thus, a role for IGF-IR in trastuzumab resistance appears to be supported by several clinical correlative studies and should be further studied in this context.

\section{Potential Mechanisms of IGF-IR-Mediated Trastuzumab Resistance}

IGF-IR consists of two alpha and two beta subunits synthesized from a single mRNA precursor. The subunits are cross-linked by cysteine bonds. The alpha chains are extracellular and form the ligand-binding domain, while the transmembrane beta regions possess intrinsic tyrosine kinase activity. IGF-IR tyrosine kinase is activated upon binding IGF-I, inducing autophosphorylation of the receptor (Figure 2). The insulin receptor substrate (IRS) and Shc adaptor proteins, which are bound to the IGF-IR beta subunits, will then become phosphorylated. These proteins couple to and activate downstream signaling through RasRaf-MEK-Erk, resulting in increased proliferation. IRS proteins also couple to PI3K, activation of which converts PIP2 to PIP3, and recruits Akt to the plasma membrane where it is activated by PDK. Activated Akt promotes proliferation, cell survival, and migration [27], in part due to activation of mTOR signaling, which increases protein translation.

The mechanisms by which IGF-IR promotes trastuzumab resistance remain largely unknown, although some studies have implicated a role for PI3K signaling. Increased PI3K signaling has been shown to reduce response to trastuzumab in multiple preclinical models [28-31], and was shown to be correlated with clinical trastuzumab resistance $[28,30$, 32]. Mechanisms resulting in increased PI3K signaling have primarily focused on downregulation of the phosphatase PTEN and hyper-activating mutations in PIK3CA; however, increased growth factor signaling, such as through IGFIR, will also result in increased PI3K signaling. IGF-I stimulation of cells with acquired trastuzumab resistance induced phosphorylation of IRS-1, HER2, Akt, and Erk1/2 with reduced expression of p27kip1 [20]. Tyrosine kinase inhibition or antibody blockade of IGF-IR blocked phosphorylation of HER2, Akt, and Erk1/2. In MCF7/HER2 and SKBR3/IGF-IR stably transfected cells, IGF-I stimulation blocked trastuzumab-mediated inhibition of Akt and Erk1/2 phosphorylation [16]. A pharmacological inhibitor of phosphoinositide-dependent kinase-1 (PDK-1), OSU03012 increased trastuzumab-mediated growth inhibition in SKBR3/IGF-IR cells through downregulation of PDK1/Akt signaling [14]. In addition to PI3K signaling, IGFIR is likely to promote resistance via activation of mTOR. Inhibition of $\mathrm{mTOR}$ has been effective in restoring sensitivity to trastuzumab in a variety of settings [33-35]. Combination trastuzumab plus rapamycin reduced colony formation and invasion in multiple HER2-positive breast cancer cell lines including those with high endogenous IGF-IR expression [36]. Interestingly, a recent clinical correlative study showed that mTOR was overexpressed by IHC in $23 \%$ of tumors from patients treated with trastuzumab, in association with IGF-IR over-expression in $47 \%$ of the tumors, p110 subunit of PIK3CA in 64\% of tumors, and phosphorylation (inactivation) of proapoptotic Bad in 65\% of tumors [26]. Thus, in almost half of the tumors that overexpressed mTOR, there was also a potential role for upregulation of IGF-IRAkt-Bad phosphorylation.

Downstream events resulting in altered expression and function of cell cycle regulators appear to mediate the ultimate increase in proliferation and cell survival propagated by increased IGF-IR signaling. Stable over-expression 
of IGF-IR resulted in reduced expression of the cyclindependent kinase inhibitors p27kip1 and p21cip1 and increased expression of cyclin E [13]. Similarly, trastuzumabresistant cells have been reported to show reduced p27kip1 [19] and increased cyclin E expression [37]. We showed that cells with acquired resistance to trastuzumab expressed reduced levels of p27kip1 protein [19] but not transcript [38]. Thus, downregulation of p27kip1 appears to occur through posttranslational mechanisms. P27kip1 is known to be largely regulated by phosphorylation and ubiquitination events. IGF-I stimulation in SKBR3/IGF-IR stable transfectants resulted in increased expression of a p27kip1 ubiquitin ligase, Skp2 [39]. We showed that the proteasome inhibitor MG132 induces p27kip1 expression in cells with acquired trastuzumab resistance back to parental levels [19]. Further, resistant cells showed increased sensitivity to MG132 versus parental cells. IGF-I was shown to increase ubiquitination of p27kip1 protein in SKBR3/IGF-IR cells [39]. These results suggest that a potential mechanism by which IGF-IR promotes trastuzumab resistance is via increased ubiquitination and downregulation of p27kip1 protein, resulting in reduced growth arrest and increased proliferation. In fact, reduced p27kip1 expression in resistant cells was associated with increased cdk2 activity and an increased fraction of cells in S phase (proliferation) [19]. Transfection of p27kip1 increased sensitivity to trastuzumab [19], suggesting that downregulation of this downstream protein is an important mechanism of resistance.

Scaltriti et al. [37] showed that amongst 34 patients with HER2-positive breast cancer, cyclin $E$ amplification and over-expression was associated with poor clinical benefit to trastuzumab $(33.3 \%$ compared with $87.5 \%$ in those without amplification) and lower progression-free survival (6 months versus 14 months). Over-expression of cyclin E was associated with higher cdk2 activity, and cdk2 inhibition reduced growth of trastuzumab-resistant cell xenografts [37]. Thus, mechanisms downstream of increased IGF-IR signaling, including reduced p27kip 1 and increased cyclin E expression, both of which result in increased cdk2 activity, have been reported in trastuzumab-resistant cells.

\section{Role of Insulin-Like Growth Factor-I-Binding Proteins (IGFBPS) in Trastuzumab Resistance}

The IGF-I signaling family includes at least 6 human IGFbinding proteins (IGFBPs). Some IGFBPs bind and sequester IGF-I such that the ligand is unable to bind and activate its receptor. Studies suggest that increased circulating IGFBP levels (particularly IGFBP3) may be used as a marker of increased IGF-IR signaling and trastuzumab resistance; others show that increased expression of IGFBP3 abrogates IGF-IR signaling and increases sensitivity to trastuzumab.

Increased expression of recombinant human IGFBP3 improved response to trastuzumab in multiple models of resistance $[13,16]$. In one study [13], MCF7/HER2 stable transfectants, which express high levels of IGF-IR, were not inhibited by trastuzumab in soft agar conditions. IGFBP3 alone inhibited growth by $29 \%$, whereas the combination of trastuzumab plus IGFBP3 inhibited growth by $82 \%$. Similarly, SKBR3/IGF-IR stable transfectants, which were resistant to trastuzumab, showed growth inhibition when cotreated with trastuzumab plus IGFBP3 [13]. Synergy between IGFBP3 and trastuzumab was confirmed by statistical analysis of drug combination dose-effects in SKBR3/IGF-IR, MCF7/HER2, and BT474 acquired resistant cells, but not in parental cells [16]. IGFBP3 suppressed IGF-I signaling in these cell line and xenograft models of resistance [13, $16,40]$. Tumor growth of MCF7/HER2 xenografts was not inhibited by single-agent trastuzumab, whereas single-agent IGFBP3 showed a trend toward growth inhibition [16]. Combined IGFBP 3 and trastuzumab treatment resulted in a statistically significant reduction in MCF7/HER2 xenograft tumor volume. IHC analysis of tumor samples showed that Akt and Erk1/2 phosphorylation was maintained at control levels in the trastuzumab-treated group, whereas IGFBP3 (alone or in combination with trastuzumab) reduced Akt and MAPK signaling.

Dokmanovic et al. [41] further suggested that elevated levels of IGFBP3 may reduce IGF-IR/HER2 crosstalk. They showed that trastuzumab induced expression and secretion of IGFBP3 and IGFBP2 in SKBR3 cells in association with growth inhibition. Increased IGFBP3 levels resulted in reduced IGF-I-mediated phosphorylation of IGF-IR, HER2, Akt, and Erk1/2. Further, cells with acquired or intrinsic resistance showed reduced levels of IGFBP3. In contrast, IGFBP2 stimulated phosphorylation of HER2, which was reduced by trastuzumab treatment. Transient transfection of an IGFBP3 expression plasmid into SKBR3 parental or acquired trastuzumab-resistant cells resulted in reduced cell viability [41].

These studies indicate that reduced expression of an endogenous negative regulator of IGF-I activity, IGFBP3, may serve as an indicator of IGF-I signaling and trastuzumab resistance. Strategies that deliver IGFBP3 as a therapy may benefit breast cancers that are resistant to trastuzumab and show elevated IGF-IR signaling.

\section{IGF-IR Inhibition as a Strategy to Improve Response to Trastuzumab}

Due to preclinical and clinical data suggesting that IGFIR signaling reduces response to trastuzumab, therapeutic strategies that co-target IGF-IR and HER2 have been studied in models of HER2-over-expressing breast cancer. We showed that the IGF-IR monoclonal antibody (mAb) alpha IR3 restored sensitivity to trastuzumab in models of acquired trastuzumab resistance, in association with disruption of IGF-IR/HER2 dimerization [20]. IGF-IR tyrosine kinase inhibitor (TKI) AG538 also produced dosedependent reductions in survival of resistant cells [20]. In contrast, trastuzumab-sensitive BT474 cells showed little response to single-agent alpha IR3 or IGF-IR TKI AG1024 [42]. However, combining these IGF-IR inhibitors with trastuzumab or HER2 kinase inhibitor AG825 resulted in synergistic growth inhibition, increased G1 arrest, reduced 
proliferation and increased apoptosis [42]. Interestingly, interaction and crosstalk between IGF-IR and HER2 were noted in BT474 cells, with IGF-IR inhibition reducing HER2 phosphorylation $[42,43]$. Cornelissen et al. [15] showed that resistance of HER2-overexpressing breast tumor xenografts to trastuzumab correlated with IGF-IR density, and that this resistance was reversed by addition of IGFBP3 or IGF-IR TKI AG1024. The IGF-IR kinase inhibitor NVP-AEW541 also achieved synergistic inhibition of proliferation when combined with trastuzumab in BT474 cells, with reduced pAkt and increased p27kip1 expression [44]. NVP-AEW541 reduced proliferation of trastuzumab-resistant BT474 and SKBR3 cells when combined with trastuzumab [43]. The phenolic compound nordihydroguaiaretic acid (NDGA) suppressed IGF-IR and HER2 signaling [45, 46], and induced cell death of trastuzumab-naive and trastuzumab-refractory HER2-over-expressing breast cancer cells [45].

Several IGF-IR-targeted agents are currently in early phases of clinical development for various types of cancer. IGF-IR mAb ganitumab (AMG 479; Amgen) has elicited responses in treatment-refractory Ewing's sarcoma [47] and is currently being studied in combination with trastuzumab in patients with HER2-positive metastatic breast cancer. Cixutumumab (IMC-A12; ImClone Systems Incorporated) treatment showed a trend toward improved progressionfree survival in patients with refractory nonsmall lung cancer who had high baseline IGF-I levels [48]. However, the combination of cixutumumab and EGFR TKI erlotinib that were tested in that study was not tolerable. Cixutumumab is now being tested in combination with lapatinib in patients with metastatic breast cancer. Assessing the dose-limiting toxicity of this combination and careful biomarker analysis will be keys to determining whether dual IGF-IR/HER2 targeting is achievable and what molecular predictors should be measured to rationally select patients to receive this combination. Dalotuzumab (MK0646, h7C10; Merck) is another IGF-IR mAb being tested in various clinical settings. Previous work showed that dalotuzumab and the EGFR mAb 225 elicited synergistic tumor regression in a xenograft model of the MCF7 breast cancer line [49]. In the context of HER2-positive breast cancer, dalotuzumab is being tested in combination with trastuzumab in xenograft models of trastuzumab resistance. Dual IGF-IR/insulin receptor TKIs BMS-754807 and BMS754807 (Bristol Myers Squibb) are also being tested in combination with trastuzumab in HER2-positive metastatic breast cancer.

\section{Conclusions}

In summary, IGF-IR expression and signaling are elevated in a subset of trastuzumab-resistant breast cancers. Inhibition of IGF-IR using genetic or pharmacologic approaches shows antitumor activity in cell lines and xenograft models derived from trastuzumab-naïve and trastuzumab-resistant HER2positive breast cancers. Further in vivo analysis of combination IGF-IR and HER2 targeting is required, as only a few studies have shown that pharmacologic inhibition of
IGF-IR restores sensitivity to trastuzumab in animal models. In addition, the mechanism of IGF-IR-mediated trastuzum$\mathrm{ab}$ resistance remains largely unknown. While PI3K signaling appears to play a role, IGF-IR activates multiple downstream pathways that are likely to also be involved in drug resistance. Identification of the signaling molecules that contribute to IGF-I-dependent trastuzumab resistance may provide biomarkers or additional therapeutic targets.

Several IGF-IR antibodies and kinase inhibitors are now being tested clinically. Based on the preclinical and clinical correlative data presented in this paper, there is rationale for conducting trials that combine IGF-IR inhibitors with trastuzumab in the context of HER2-over-expressing breast cancer that has progressed on prior trastuzumabcontaining regimens. Careful biomarker analysis will be an important part of these trials. Therapeutic strategies that co-target IGF-IR and HER2 are likely to achieve benefit in only a subset of trastuzumab-refractory disease, since IGF-independent mechanisms of resistance are also known to occur. In the absence of rational patient selection for trials of IGF-IR-directed agents, a clinical benefit in a subpopulation may be missed. Thus, trastuzumab-refractory breast cancer tissue or patient serum should be examined for possible predictors of response to IGF-IR therapy, such as increased IGF-I levels, reduced IGFBP3 levels, or increased expression or phosphorylation of IGF-IR. Serum markers such as IGF-I or IGFBPs would be easier to obtain, as most patients with metastatic, trastuzumab-refractory disease do not return for biopsy. Validation of these serum markers as true indicators of response will help achieve this important step of rationally selecting which patients should be treated with combination IGF-IR inhibitors plus trastuzumab.

Strong preclinical data implicating increased IGF-IR signaling as a mechanism of trastuzumab resistance has been collected over the past decade. The next 3-5 years will help to establish whether IGF-IR signaling is a valid clinical predictor of trastuzumab resistance, and/or if IGF-IR is a molecular target whose inhibition can improve response to trastuzumab in patients with HER2-overexpressing metastatic breast cancer.

\section{Support}

National Cancer Institute (K01CA118174 and 3K01CA118174-5S1), Georgia Cancer Coalition Distinguished Cancer Scholar Award, P30 CA138292 (Winship Cancer Institute).

\section{References}

[1] P. Carter, L. Presta, C. M. Gorman et al., "Humanization of an anti-p185(HER2) antibody for human cancer therapy," Proceedings of the National Academy of Sciences of the United States of America, vol. 89, no. 10, pp. 4285-4289, 1992.

[2] D. J. Slamon, G. M. Clark, S. G. Wong, W. J. Levin, A. Ullrich, and W. L. McGuire, "Human breast cancer: correlation of relapse and survival with amplification of the HER-2/neu oncogene," Science, vol. 235, no. 4785, pp. 177-182, 1987. 
[3] J. Baselga, D. Tripathy, J. Mendelsohn et al., "Phase II study of weekly intravenous recombinant humanized anti-p185HER2 monoclonal antibody in patients with HER2/neu-overexpressing metastatic breast cancer," Journal of Clinical Oncology, vol. 14, no. 3, pp. 737-744, 1996.

[4] M. A. Cobleigh, C. L. Vogel, D. Tripathy et al., "Multinational study of the efficacy and safety of humanized antiHER2 monoclonal antibody in women who have HER2overexpressing metastatic breast cancer that has progressed after chemotherapy for metastatic disease," Journal of Clinical Oncology, vol. 17, no. 9, pp. 2639-2648, 1999.

[5] F. J. Esteva, V. Valero, D. Booser et al., "Phase II study of weekly docetaxel and trastuzumab for patients with HER-2-overexpressing metastatic breast cancer," Journal of Clinical Oncology, vol. 20, no. 7, pp. 1800-1808, 2002.

[6] A. D. Seidman, M. N. Fornier, F. J. Esteva et al., "Weekly trastuzumab and paclitaxel therapy for metastatic breast cancer with analysis of efficacy by HER2 immunophenotype and gene amplification," Journal of Clinical Oncology, vol. 19, no. 10, pp. 2587-2595, 2001.

[7] D. J. Slamon, B. Leyland-Jones, S. Shak et al., "Use of chemotherapy plus a monoclonal antibody against her2 for metastatic breast cancer that overexpresses HER2," New England Journal of Medicine, vol. 344, no. 11, pp. 783-792, 2001.

[8] C. L. Arteaga, M. X. Sliwkowski, C. K. Osborne, E. A. Perez, F. Puglisi, and L. Gianni, "Treatment of HER2-positive breast cancer: current status and future perspectives," Nature Reviews Clinical Oncology, vol. 9, pp. 16-32, 2012.

[9] T. A. Bailey, H. Luan, R. J. Clubb, M. Naramura, V. Band, S. M. Raja et al., "Mechanisms of Trastuzumab resistance in ErbB2driven breast cancer and newer opportunities to overcome therapy resistance," Journal of Carcinogenesis, vol. 10, article $28,2011$.

[10] R. Nahta, "Pharmacological strategies to overcome HER2 cross-talk and Trastuzumab resistance," Current Medicinal Chemistry, vol. 19, no. 7, pp. 1065-1075, 2012.

[11] R. Nahta, D. Yu, M. C. Hung, G. N. Hortobagyi, and F. J. Esteva, "Mechanisms of disease: understanding resistance to HER2-targeted therapy in human breast cancer," Nature Clinical Practice Oncology, vol. 3, no. 5, pp. 269-280, 2006.

[12] R. Y. Tsang and R. S. Finn, "HER2-positive breast cancer: trastuzumab, lapatinib and emerging therapies," Drug Discovery Today: Therapeutic Strategies, vol. 106, pp. 6-13, 2012.

[13] Y. Lu, X. Zi, Y. Zhao, D. Mascarenhas, and M. Pollak, "Insulin-like growth factor-I receptor signaling and resistance to transtuzumab (Herceptin)," Journal of the National Cancer Institute, vol. 93, no. 24, pp. 1852-1857, 2001.

[14] P. H. Tseng, Y. C. Wang, S. C. Weng et al., "Overcoming trastuzumab resistance in HER2-overexpressing breast cancer cells by using a novel celecoxib-derived phosphoinositidedependent kinase-1 inhibitor," Molecular Pharmacology, vol. 70, no. 5, pp. 1534-1541, 2006.

[15] B. Cornelissen, K. McLarty, V. Kersemans, and R. M. Reilly, "The level of insulin growth factor-1 receptor expression is directly correlated with the tumor uptake of 111In-IGF1(E3R) in vivo and the clonogenic survival of breast cancer cells exposed in vitro to trastuzumab (Herceptin)," Nuclear Medicine and Biology, vol. 35, no. 6, pp. 645-653, 2008.

[16] L. Jerome, N. Alami, S. Belanger et al., "Recombinant human insulin-like growth factor binding protein 3 inhibits growth of human epidermal growth factor receptor-2-overexpressing breast tumors and potentiates herceptin activity in vivo," Cancer Research, vol. 66, no. 14, pp. 7245-7252, 2006.
[17] C. Oliveras-Ferraros, A. Vazquez-Martin, B. Martin-Castillo et al., "Pathway-focused proteomic signatures in HER2overexpressing breast cancer with a basal-like phenotype: new insights into de novo resistance to trastuzumab (Herceptin)," International Journal of Oncology, vol. 37, no. 3, pp. 669-678, 2010.

[18] R. Nahta and F. J. Esteva, "In vitro effects of trastuzumab and vinorelbine in trastuzumab-resistant breast cancer cells," Cancer Chemotherapy and Pharmacology, vol. 53, no. 2, pp. 186-190, 2004.

[19] R. Nahta, T. Takahashi, N. T. Ueno, M. C. Hung, and F. J. Esteva, "P27kip1 down-regulation is associated with trastuzumab resistance in breast cancer cells," Cancer Research, vol. 64, no. 11, pp. 3981-3986, 2004.

[20] R. Nahta, L. X. H. Yuan, B. Zhang, R. Kobayashi, and F. J. Esteva, "Insulin-like growth factor-I receptor/human epidermal growth factor receptor 2 heterodimerization contributes to trastuzumab resistance of breast cancer cells," Cancer Research, vol. 65, pp. 11118-11128, 2005.

[21] X. Huang, L. Gao, S. Wang et al., "Heterotrimerization of the growth factor receptors erbB2, erbB3, and insulin-like growth factor-I receptor in breast cancer cells resistant to herceptin," Cancer Research, vol. 70, no. 3, pp. 1204-1214, 2010.

[22] W. J. Köstler, G. Hudelist, W. Rabitsch et al., "Insulin-like growth factor-1 receptor (IGF-1R) expression does not predict for resistance to trastuzumab-based treatment in patients with Her-2/neu overexpressing metastatic breast cancer," Journal of Cancer Research and Clinical Oncology, vol. 132, no. 1, pp. 918, 2006.

[23] B. L. Smith, D. Chin, W. Maltzman, K. Crosby, G. N. Hortobagyi, and S. S. Bacus, "The efficacy of Herceptin therapies is influenced by the expression of other erbB receptors, their ligands and the activation of downstream signalling proteins," British Journal of Cancer, vol. 91, no. 6, pp. 1190-1194, 2004.

[24] R. Yerushalmi, K. A. Gelmon, S. Leung et al., "Insulin-like growth factor receptor (IGF-1R) in breast cancer subtypes," Breast Cancer Research and Treatment, vol. 132, pp. 131-142, 2012.

[25] L. N. Harris, F. You, S. J. Schnitt et al., "Predictors of resistance to preoperative trastuzumab and vinorelbine for HER2positive early breast cancer," Clinical Cancer Research, vol. 13, no. 4, pp. 1198-1207, 2007.

[26] A. Gallardo, E. Lerma, D. Escuin, A. Tibau, J. Munoz, B. Ojeda et al., "Increased signalling of EGFR and IGF1R, and deregulation of PTEN/PI3K/Akt pathway are related with trastuzumab resistance in HER2 breast carcinomas," British Journal of Cancer, vol. 106, pp. 1367-1373, 2012.

[27] H. Zhang and D. Yee, "Is the type I insulin-like growth factor receptor a therapeutic target in endometrial cancer?" Clinical Cancer Research, vol. 12, no. 21, pp. 6323-6325, 2006.

[28] K. Berns, H. M. Horlings, B. T. Hennessy et al., "A functional genetic approach identifies the PI3K pathway as a major determinant of Trastuzumab resistance in breast cancer," Cancer Cell, vol. 12, no. 4, pp. 395-402, 2007.

[29] C. H. Lu, S. L. Wyszomierski, L. M. Tseng et al., "Preclinical testing of clinically applicable strategies for overcoming trastuzumab resistance caused by PTEN deficiency," Clinical Cancer Research, vol. 13, no. 19, pp. 5883-5888, 2007.

[30] Y. Nagata, K. H. Lan, X. Zhou et al., "PTEN activation contributes to tumor inhibition by trastuzumab, and loss of PTEN predicts trastuzumab resistance in patients," Cancer Cell, vol. 6, no. 2, pp. 117-127, 2004.

[31] T. Ozbay, D. L. Durden, T. Liu, R. M. O’Regan, and R. Nahta, "In vitro evaluation of pan-PI3-kinase inhibitor SF1126 
in trastuzumab-sensitive and trastuzumab-resistant HER2over-expressing breast cancer cells," Cancer Chemotherapy and Pharmacology, vol. 65, no. 4, pp. 697-706, 2010.

[32] F. J. Esteva, H. Guo, S. Zhang et al., "PTEN, PIK3CA, p-AKT, and p-p70S6K status: association with trastuzumab response and survival in patients with HER2-positive metastatic breast cancer," American Journal of Pathology, vol. 177, no. 4, pp. 1647-1656, 2010.

[33] F. Andre, M. Campone, R. O’Regan et al., "Phase I study of everolimus plus weekly paclitaxel and Trastuzumab in patients with metastatic breast cancer pretreated with Trastuzumab," Journal of Clinical Oncology, vol. 28, no. 34, pp. 5110-5115, 2010.

[34] S. S. Gayle, S. L. Arnold, R. M. O’Regan, and R. Nahta, "Pharmacologic inhibition of mTOR improves lapatinib sensitivity in HER2-overexpressing breast cancer cells with primary Trastuzumab resistance," Anti-Cancer Agents in Medicinal Chemistry, vol. 12, pp. 151-162, 2012.

[35] P. K. Morrow, G. M. Wulf, J. Ensor, D. J. Booser, J. A. Moore, P. R. Flores et al., "Phase I/II study of trastuzumab in combination with everolimus (RAD001) in patients with HER2overexpressing metastatic breast cancer who progressed on trastuzumab-based therapy," Journal of Clinical Oncology, vol. 29, pp. 3126-3132, 2011.

[36] L. H. Wang, J. L. K. Chan, and W. Li, "Rapamycin together with herceptin significantly increased anti-tumor efficacy compared to either alone in ErbB2 over expressing breast cancer cells," International Journal of Cancer, vol. 121, no. 1, pp. 157-164, 2007.

[37] M. Scaltriti, P. J. Eichhorn, J. Cortés et al., "Cyclin E amplification/overexpression is a mechanism of trastuzumab resistance in HER2 ${ }^{+}$breast cancer patients," Proceedings of the National Academy of Sciences of the United States of America, vol. 108, no. 9, pp. 3761-3766, 2011.

[38] S. Shabaya and R. Nahta, "Novel therapeutic strategies and combinations for HER2-overexpressing breast cancer," in Breast Cancer-Current and Alternative Therapeutic Modalities, E. Gunduz and M. Gunduz, Eds., pp. 3-22, InTech, 2011.

[39] Y. Lu, X. Zi, and M. Pollak, "Molecular mechanisms underlying IGF-I-induced attenuation of the growth-inhibitory activity of trastuzumab (herceptin) on SKBR3 breast cancer cells," International Journal of Cancer, vol. 108, no. 3, pp. 334341, 2004.

[40] Y. Lu, X. Zi, Y. Zhao, and M. Pollak, “Overexpression of ErbB2 receptor inhibits IGF-I-induced Shc-MAPK signaling pathway in breast cancer cells," Biochemical and Biophysical Research Communications, vol. 313, no. 3, pp. 709-715, 2004.

[41] M. Dokmanovic, Y. Shen, T. M. Bonacci, D. S. Hirsch, and W. J. Wu, "Trastuzumab regulates IGFBP-2 and IGFBP-3 to mediate growth inhibition: implications for the development of predictive biomarkers for trastuzumab resistance," Molecular Cancer Therapeutics, vol. 10, no. 6, pp. 917-928, 2011.

[42] A. K. Chakraborty, K. Liang, and M. P. DiGiovanna, "Cotargeting insulin-like growth factor I receptor and HER2: dramatic effects of HER2 inhibitors on nonoverexpressing breast cancer," Cancer Research, vol. 68, no. 5, pp. 1538-1545, 2008.

[43] B. C. Browne, J. Crown, N. Venkatesan et al., "Inhibition of IGF1R activity enhances response to trastuzumab in HER-2positive breast cancer cells," Annals of Oncology, vol. 22, no. 1, pp. 68-73, 2011.

[44] A. Esparís-Ogando, A. Ocaña, R. Rodríguez-Barrueco, L. Ferreira, J. Borges, and A. Pandiella, "Synergic antitumoral effect of an IGF-IR inhibitor and trastuzumab on HER2overexpressing breast cancer cells," Annals of Oncology, vol. 19, no. 11, pp. 1860-1869, 2008.

[45] D. L. Rowe, T. Ozbay, L. M. Bender, and R. Nahta, "Nordihydroguaiaretic acid, a cytotoxic insulin-like growth factorI receptor/HER2 inhibitor in trastuzumab-resistant breast cancer," Molecular Cancer Therapeutics, vol. 7, no. 7, pp. 19001908, 2008.

[46] M. Zavodovskaya, M. J. Campbell, B. A. Maddux et al., "Nordihydroguaiaretic acid (NDGA), an inhibitor of the HER2 and IGF-1 receptor tyrosine kinases, blocks the growth of HER2overexpressing human breast cancer cells," Journal of Cellular Biochemistry, vol. 103, no. 2, pp. 624-635, 2008.

[47] W. D. Tap, G. Demetri, P. Barnette, J. Desai, P. Kavan, R. Tozer et al., "Phase II study of ganitumab, a fully human anti-type-1 insulin-like growth factor receptor antibody, in patients with metastatic ewing family tumors or desmoplastic small round cell tumors," Journal of Clinical Oncology, vol. 30, pp. 18491856, 2012.

[48] A. Weickhardt, R. Doebele, A. Oton, J. Lettieri, D. Maxson, M. Reynolds et al., "A phase I/II study of erlotinib in combination with the anti-insulin-like growth factor-1 receptor monoclonal antibody IMC-A12 (cixutumumab) in patients with advanced non-small cell lung cancer," Journal of Thoracic Oncology, vol. 7, pp. 419-426, 2012.

[49] L. Goetsch, A. Gonzalez, O. Leger et al., "A recombinant humanized anti-insulin-like growth factor-receptor type I antibody (h7C10) enhances the antitumor activity of vinorelbine and anti-epidermal growth factor receptor therapy against human cancer xenografts," International Journal of Cancer, vol. 113, no. 2, pp. 316-328, 2005. 


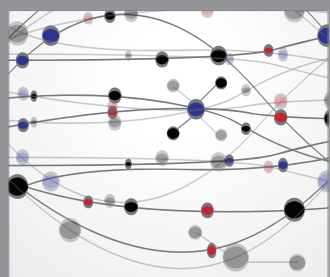

The Scientific World Journal
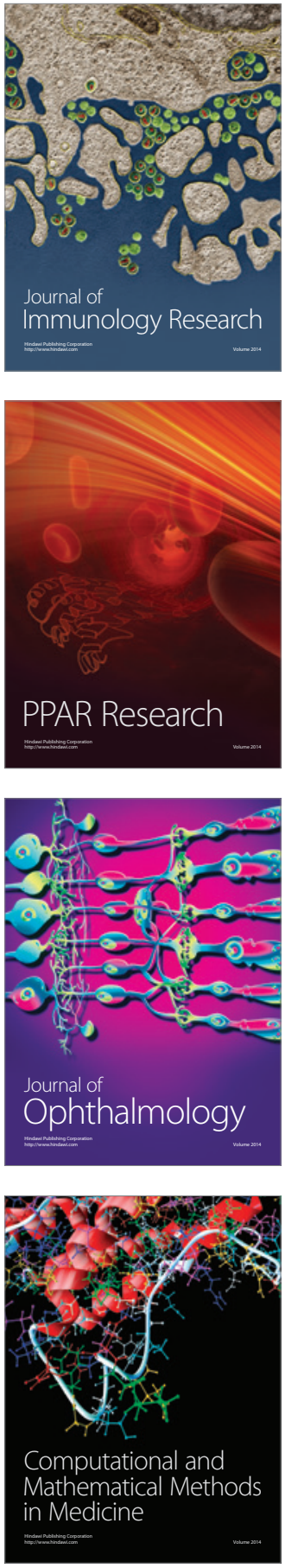

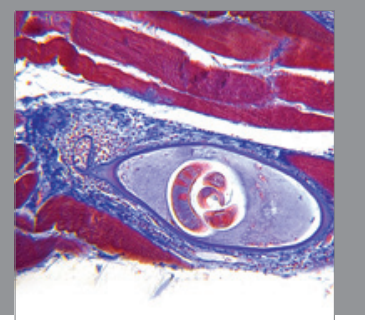

Gastroenterology

Research and Practice
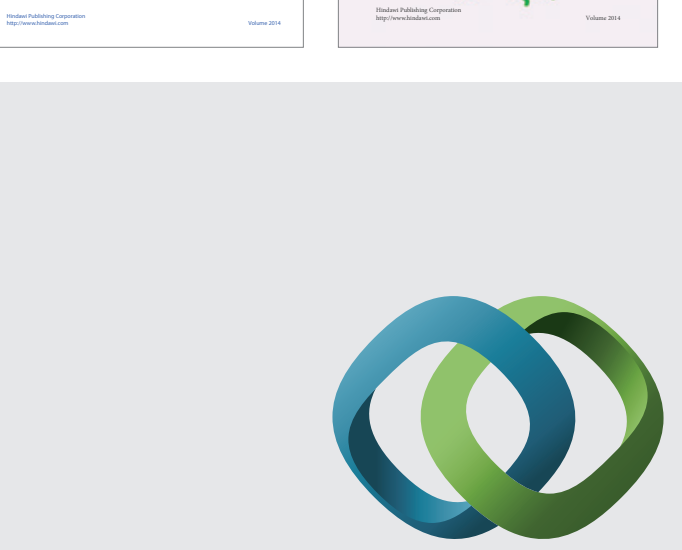

\section{Hindawi}

Submit your manuscripts at

http://www.hindawi.com
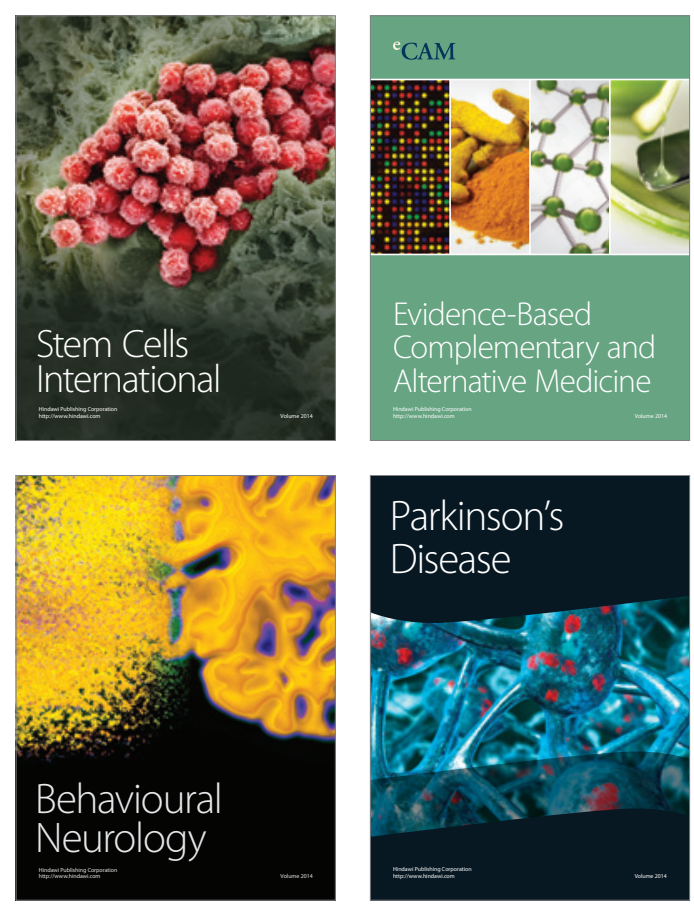

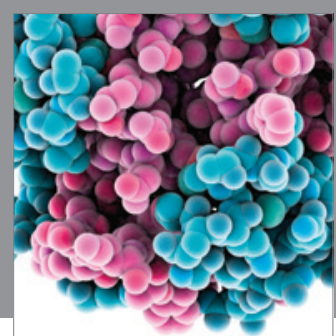

Journal of
Diabetes Research

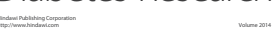

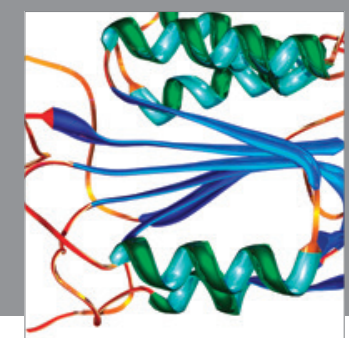

Disease Markers
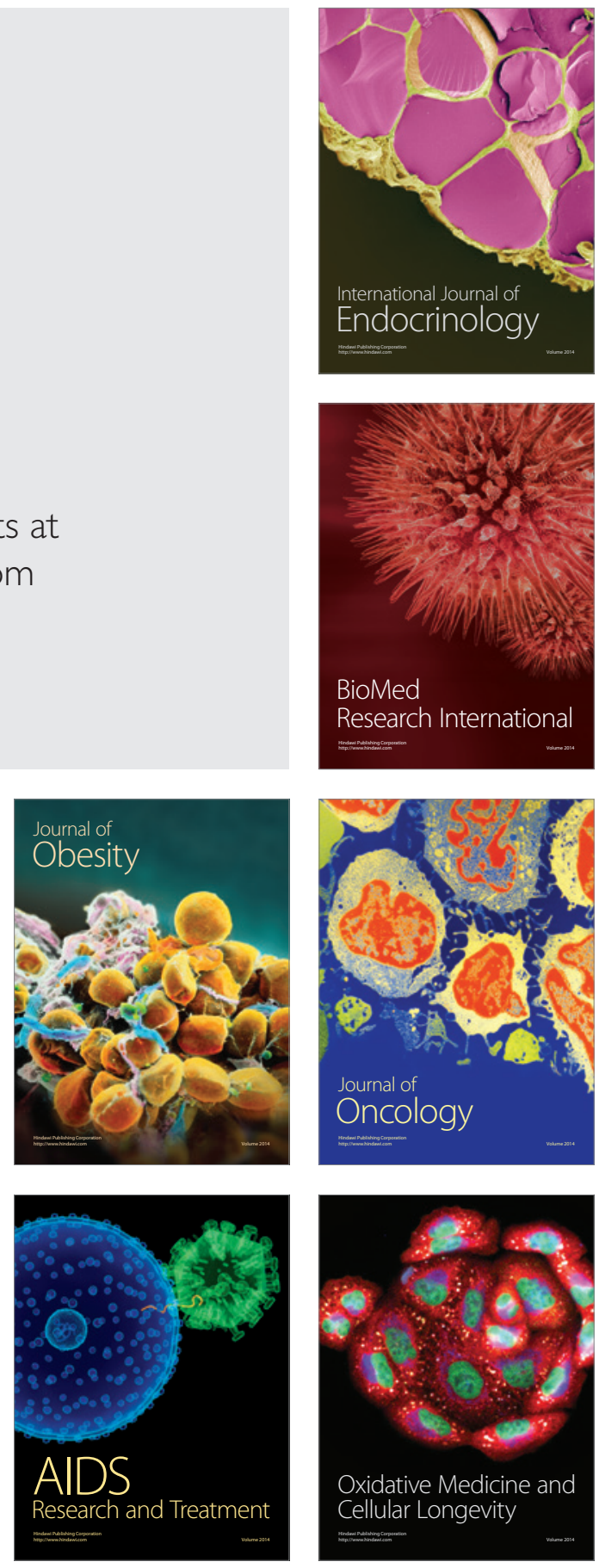